\title{
Three-Dimensional Slice Image Overlay System with Accurate Depth Perception for Surgery
}

\author{
Ken Masamune,Yoshitaka Masutani*,Susumu Nakajima+,Ichiro Sakuma**, \\ Takeyoshi Dohi**, Hiroshi Iseki*** and Kintomo Takakura*** \\ Dept. of Biotechnology, Tokyo Denki University \\ *Graduate school of Engineering, the University of Tokyo, \\ **Graduate school of Frontier Science, the University of Tokyo \\ +Dept. of Orthopedic surgery, the University of Tokyo \\ ***Dept. of Neurosurgery, Tokyo Women's Medical University \\ Ishizaka, Hatoyama, Hiki, Saitama, 350-0394 Japan \\ masa@b.dendai.ac.jp
}

\begin{abstract}
In this paper, we describe a three-dimensional (3-D) display, containing a flat two-dimensional (2-D) display, an actuator and a half-silvered mirror. This system creates a superimposed slice view on the patient and gives accurate depth perception. The clinical significance of this system is that it displays raw image data at an accurate location on the patient's body. Moreover, it shows previously acquired image information, giving the capacity for accurate direction to the surgeon who is thus able to perform less-invasive therapy. Compared with conventional 3-D displays, such as stereoscopy, this system only requires raw 3-D data that are acquired in advance. Simpler data processing is required, and the system has the potential for rapid development. We describe a novel algorithm, registering positional data between the image and the patient. The accuracy of the system is evaluated and confirmed by an experiment in which an image is superimposed on a test object. The results indicate that the system could be readily applied in clinical situations, considering the resolution of the pre-acquired images.
\end{abstract}

\section{Introduction}

Recently, many researches described the use of three-dimensional (3-D) medical images for surgical simulation and planning, before and after surgery from the end of 1980's[1]. Ordinarily, these 3-D medical images are shown on the flat twodimensional (2-D) monitor of a computer. In general, 3-D images play an important role in allowing the surgeon to recognize the region of interest and to coordinate the spatial relationships between the patient and the images. However, surgeons are constrained by having to watch a display in a corner of the operating room. Thus, their actions are not truly objective and it is not possible to register the images with the patient in an accurate manner. This problem is particularly acute for 2-D slice images.

To increase comprehension of pre-acquired images, 3-D displays are becoming more popular in the medical field, with various modes of visualization on offer. The most popular method of observing such images is to use the principle of stereoscopic display, for example, using a polarized shuttering system or reticular lens system.

S.L. Delp, A.M. DiGioia, and B. Jaramaz (Eds.): MICCAI 2000, LNCS 1935, pp. 395-402, 2000.

(C) Springer-Verlag Berlin Heidelberg 2000 
Such 3-D display systems are very convenient in the operating theater, especially in the field of Virtual Reality (VR).

However, there are problems in the use of binocular stereoscopic methodology, especially from the point of registration of 3-D medical images. In a stereoscopic 3-D display, we normally use two images: right and left. Thus, the surgeon looks at the right image with the right eye and at left image with the left eye. In general, we continuously tune our eyes' convergence and focus to recognize 3-D objects. In the case of stereoscopic displays, we can only observe pre-fixed 2-D images, which create quasi 3-D images with distortion. Subjectively we perceive it as 3-D, but there may be significant inaccuracies in registration. Thus, stereoscopic displays are not good enough for surgery, where accurate registration of image and subject is essential.

To overcome the above problems, we have developed a novel 3-D display called 'Slice display', which contains one flat 2-D display, an actuator and a half-silvered mirror. Compared with conventional 3-D displays, this system is so simple that it needs less heavy computation in image processing and has the potential for rapid development with an easy method of image-object registration. In Section 2, we describe the basic requirements for 3-D displays in surgery. The system we have developed is described in Section 3. Section 4 describes and defines the accuracy of the method of registration, which is the most important practical element. Phantom testing was performed to evaluate not only the mechanical accuracy, but also the depth perception of this system.

\section{Basic Requirements of 3-D Slice Displays for Surgery}

Three-dimensional displays will provide the most valuable information for surgeons if they meet the following conditions:

1. The surgeon should be able to recognize the 3-D position of any surgical devices on the pre-operative images with accuracy.

2. Image quality should be high.

3. There should be minimal eyestrain and fatigue during long surgical procedures.

4. The surgeon should be able to observe the neighborhood of the diseased target in 3-D.

5. Set-up, registration and sterilization of equipment should be straightforward.

Of course, the actual content and quality of the 3-D image should also be considered. The above conditions are basic points to apply in the operating theater. In particular, we put great emphasis on conditions 1, 2 and 3. And more, displaying a slice is considered clinically significant as a 3-D visualization addressed in [2].

\section{System Description [3]}

Our technique of slice-display has one degree of freedom of movement. Its principle is shown in Fig. 1. In this case, the surgeon can see the patient's head directly. In addition, the reflected image of the flat 2-D display set is visible above the patient in 
the half-silvered mirror. Finally, the surgeon can observe 2-D slice image data apparently floating "inside" the patient's head. By moving the 2-D display in the direction of the line of vision (Fig. 1), the virtual floating image is also moved in the same direction. We put a linear sensor on the 2-D display to measure the display's location. Using the measured value, we can calculate the appropriate slice image, once registration is performed. The registration method is described below. This floating 2-D slice is located relative to the 2-D display, so that the surgeon always has the correct view through the half-silvered mirror and the image will follow actual operating movements. Thus, it provides a natural view, minimizing fatigue during the operation.

Fig. 2 is the inside view of the Volume display. We use an AC motor (15 W, $100 \mathrm{~V})$ and a ball screw shaft to actuate the 2-D display. This is a 13.3-inch Super TFT Color LCD flat display screen (Selectop, Hitachi Co. Ltd., Japan). The linear encoder measures increments of $0.01 \mathrm{~mm}$. A computer (Apple Macintosh LCIII: 32MB RAM) is used to create and display the slices. The maximum size of the system is $1,000 \times$ $340 \times 390 \mathrm{~mm}$ and its weight is $12 \mathrm{~kg}$.
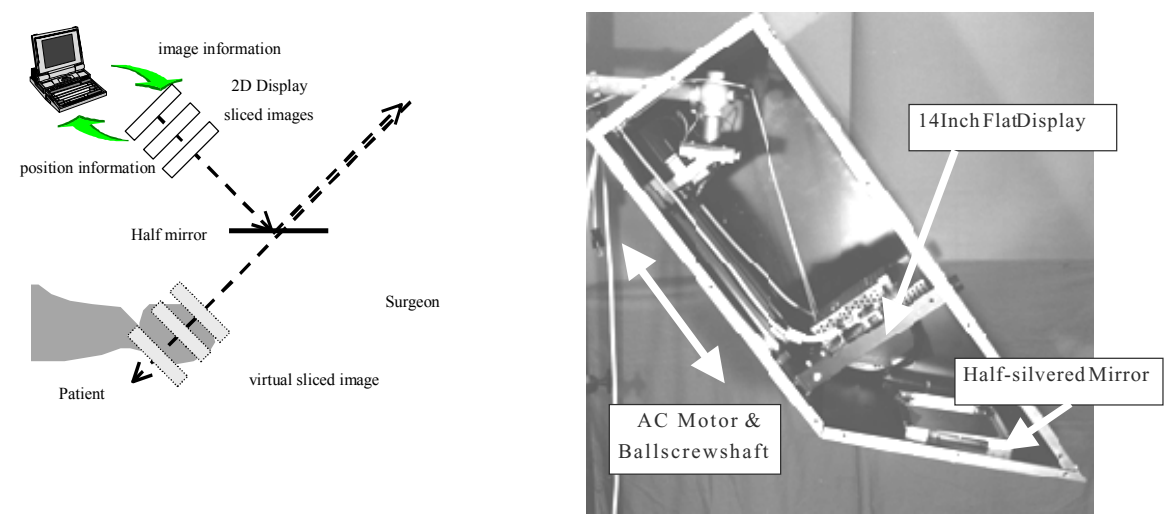

Fig. 1. Principle of Slice display;

Fig.2 Inside the Slice display.

\section{Registration}

Registration is the most important problem for display. Although many registration methods have been studied [4][5], most methods are inconvenient for practical use because they usually require complicated instruments, procedures and calculation. Considering clinical use, we applied a simple rigid matching using fiducial markers on the patients and in the images. A flow chart of the registration system is shown in Fig. 2. The fiducial marker was a triangular board and three cones shown on Fig. 3. As markers, we used capsules containing liquid Vitamin E, to enhance resolution in magnetic resonance imaging (MRI) images. The procedure was as follows:

1. Three fiducial markers were placed on the patient's head; 3-D images were obtained and converted for left-right image direction (mirroring). 
2. Three markers were identified in the 3-D images.

3. A new slice was generated, containing three markers simultaneously. This image was called the "fiducial slice image".

4. Re-slicing was carried out perpendicular to the fiducial slice image.

5. The floating image was adjusted to the real image using three markers and the surgeon's own perception.

After registration, we could observe a series of sliced images and relate them to the correct positions. All the above procedures were executed by a macro in "NIH-Image" software on the computer [6][7]. Display position was measured by linear digital scale and the data were transferred from the multiplexer to the computer by RS232C at a speed of $1200 \mathrm{bps}$.

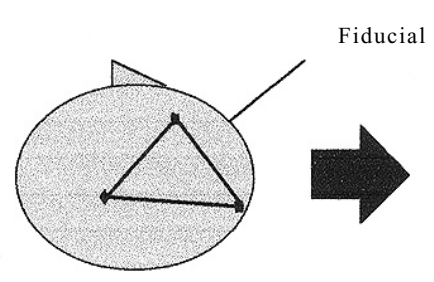

(1) 3D image from X-ray CT or MRI

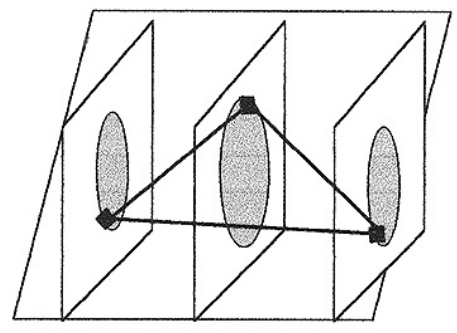

(2) Marker Detection

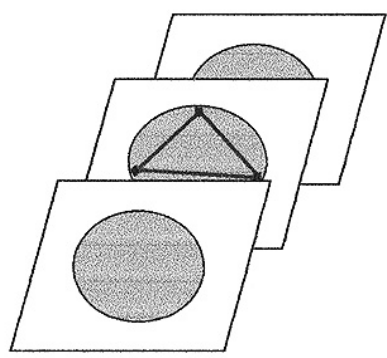

(4) Re-slicing perpendicularly to the fiducial slice image
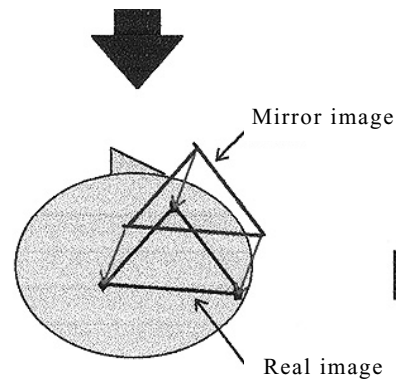

(5) Registration

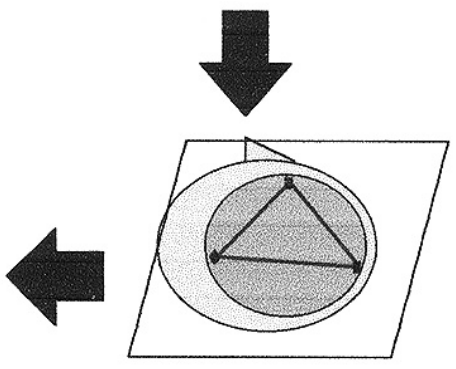

(3) Create fiducial slice image with fiducial markers

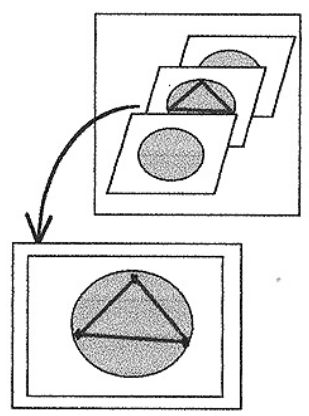

Continuing display

Fig. 3. Flow chart of the registration system. 

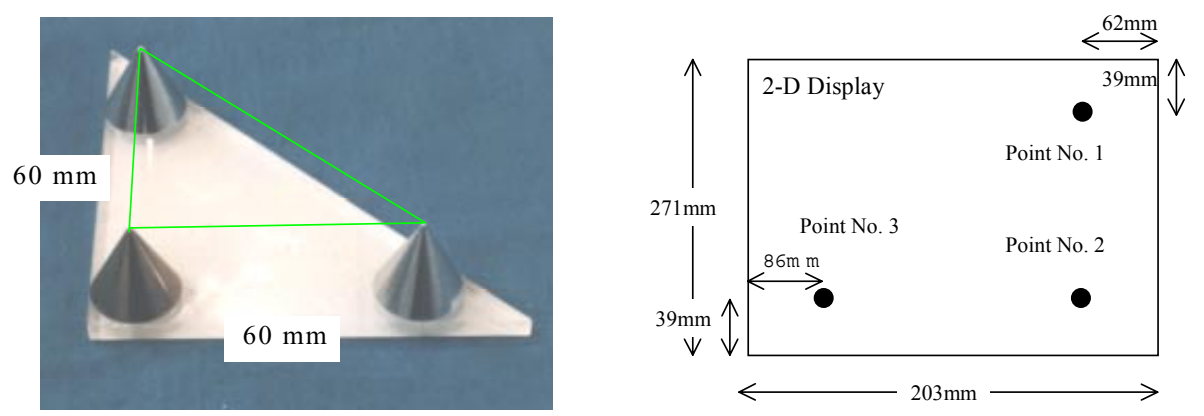

Fig.4 Triangular marker for registration. Fig. 5 Three points measured on the display.

\section{Evaluation of Accuracy}

We evaluated the mechanical and depth perception accuracy of the system. The latter factor is important because, even if we produce an accurate system, we will still have errors in the surgeon's perception of depth.

\section{Mechanical Accuracy}

Firstly, the mechanical accuracy was evaluated. The 2-D display was moved by 50$\mathrm{mm}$ increments, and three points on the display (Fig. 5) were measured in Table 1., using a 3-D digitizer (Micro Scribe 3-D,Immersion company, U.S.A.), with an accuracy of less than $0.51 \mathrm{~mm}$.

Table 1 Measured coordinate values of three points.

\begin{tabular}{|l|c|c|c|c|c|c|c|c|}
\hline \multicolumn{2}{|c|}{ Position of 2D Display } & $0.00(\mathrm{~mm})$ & $50.00(\mathrm{~mm})$ & $100.00(\mathrm{~mm})$ & $150.00(\mathrm{~mm})$ & $200.00(\mathrm{~mm})$ & $250.00(\mathrm{~mm})$ & $300.00(\mathrm{~mm})$ \\
\hline \multirow{3}{*}{ Point No.1 } & $\mathrm{x}(\mathrm{mm})$ & 33.07 & 31.02 & 28.99 & 26.94 & 24.94 & 22.89 & 20.88 \\
\cline { 2 - 9 } & $\mathrm{y}(\mathrm{mm})$ & -21.06 & -23.33 & -285.58 & -27.82 & -30.09 & -32.33 & -34.59 \\
\cline { 2 - 9 } & $\mathrm{z}(\mathrm{mm})$ & 34.15 & 38.1 & 42.15 & 46.13 & 50.12 & 54.12 & 58.05 \\
\hline \multirow{4}{*}{ Point No.2 } & $\mathrm{x}(\mathrm{mm})$ & 25.9 & 23.86 & 21.79 & 19.74 & 17.72 & 15.65 & 13.65 \\
\cline { 2 - 9 } & $\mathrm{y}(\mathrm{mm})$ & -28.72 & -30.95 & -33.17 & -35.45 & -37.72 & -39.89 & -42.18 \\
\cline { 2 - 9 } & $\mathrm{z}(\mathrm{mm})$ & 26.36 & 30.35 & 34.37 & 38.35 & 42.31 & 46.3 & 50.22 \\
\hline \multirow{4}{*}{ Point No.3 } & $\mathrm{x}(\mathrm{mm})$ & 35.34 & 33.32 & 31.3 & 29.27 & 27.22 & 25.19 & 23.18 \\
\cline { 2 - 9 } & $\mathrm{y}(\mathrm{mm})$ & -37.6 & -39.8 & -42.13 & -44.39 & -46.6 & -48.91 & -51.15 \\
\cline { 2 - 8 } & $\mathrm{z}(\mathrm{mm})$ & 26.29 & 30.3 & 34.31 & 38.28 & 42.26 & 46.21 & 50.18 \\
\hline
\end{tabular}

From these results, the maximum distortion of the display is $0.39 \mathrm{~mm}$, as seen at 300 $\mathrm{mm}$. This value is less than the error of the 3-D digitizer, and it will thus not be a significant source of error in this system.

\section{Accuracy of 3-D Perception}

In the next stage, the accuracy of 3-D perception using this system was evaluated. We prepared three points on the 2-D display as described above. Nine volunteers 
attempted to point the floating display image in the vacant space under the display system. We first measured human trembling while pointing the $3-\mathrm{D}$ digitizer within one second, and the results are shown in Table 2. The trembling error was not depend on the position of the display. Considering the accuracy of the 3 -D digitizer $(0.51$ $\mathrm{mm}$ ), this indicated that human trembling would not significantly influence the positioning procedure during the evaluation.

Table 2. Measurement of hand trembling during positioning.

\begin{tabular}{|c|c|c|c|c|c|}
\hline Display position & & Average & Maximum & Minimum & SD \\
\hline \multirow{3}{*}{0} & Point No.1 & 0.13 & 0.35 & 0.02 & 0.052 \\
\cline { 2 - 6 } & Point No.2 & 0.14 & 0.37 & 0.02 & 0.052 \\
\cline { 2 - 6 } & Point No.3 & 0.13 & 0.34 & 0.04 & 0.047 \\
\hline \multirow{3}{*}{100} & Point No.1 & 0.12 & 0.37 & 0.02 & 0.052 \\
\cline { 2 - 6 } & Point No.2 & 0.13 & 0.35 & 0.01 & 0.050 \\
\cline { 2 - 6 } & Point No.3 & 0.12 & 0.34 & 0.03 & 0.049 \\
\hline
\end{tabular}

$(\mathrm{mm})$

For evaluation, three positions of the display were measured 10 times, and the difference between the actual and theoretical point was measured and calculated.

Fig. 6 shows the positioning task by volunteer. Table 3 shows the average error for each volunteer sorted by result.

Table 3. Average positioning error.

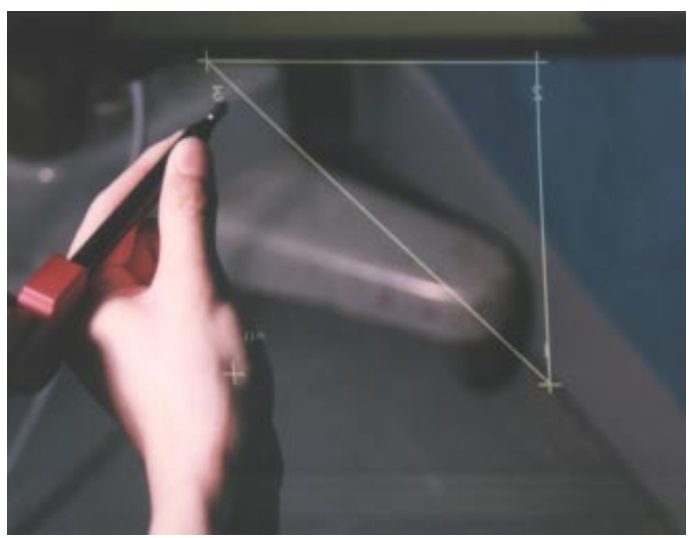

\begin{tabular}{|c|c|c|}
\hline & Average & SD \\
\hline Average & 2.13 & 1.671 \\
\hline Volunteer 1 & 0.98 & 0.221 \\
\hline Volunteer 2 & 1.03 & 0.142 \\
\hline Volunteer 3 & 1.06 & 0.139 \\
\hline Volunteer 4 & 1.08 & 0.141 \\
\hline Volunteer 5 & 1.09 & 0.130 \\
\hline Volunteer 6 & 1.43 & 0.567 \\
\hline Volunteer 7 & 3.25 & 2.145 \\
\hline Volunteer 8 & 3.83 & 2.580 \\
\hline Volunteer 9 & 5.43 & 3.793 \\
\hline
\end{tabular}

Fig. 6. Pointing testing using the 3-D digitizer.

We evaluated whether the display position depends on pointing accuracy. Table 4 and the Fig. 7. show the results. "Display position" means the distance between the first position and measuring position of the display. It increases with the distance between the surgeon's eye and the floating 2-D slice display. Most volunteers were able to point within an accuracy of $1 \mathrm{~mm}$. Considering the resolution of MRI, we thus appear to have sufficient accuracy for clinical use. However, some volunteers had much greater errors; over $10 \mathrm{~mm}$ when the display was most displaced. This error arose mainly from using 2-D slice images and the human eye. If we present the surgeon 
with a 3-D volume data set, this human error will be reduced because there will be one more degree of freedom of movement to perceive three-dimensional space.

Table 4. Pointing accuracy testing.

\begin{tabular}{|c|cc|cc|cc|}
\hline & \multicolumn{2}{|c|}{0} & \multicolumn{2}{c|}{100} & \multicolumn{2}{c|}{200} \\
& Ave. & SD & Ave. & SD & Ave. & SD \\
\hline Average & 1.21 & 0.19 & 2.4 & 1.82 & 4.08 & 4.16 \\
Vol. 1 & 1.17 & 0.11 & 1.03 & 0.08 & 0.26 & 0.10 \\
Vol. 2 & 1.11 & 0.10 & 1.04 & 0.13 & 0.76 & 0.07 \\
Vol. 3 & 1.15 & 0.11 & 1.05 & 0.12 & 0.83 & 0.12 \\
Vol. 4 & 1.18 & 0.12 & 1.07 & 0.10 & 0.81 & 0.08 \\
Vol. 5 & 1.12 & 0.11 & 1.12 & 0.12 & 0.9 & 0.07 \\
Vol. 6 & 1.24 & 0.26 & 1.17 & 0.21 & 2.81 & 1.85 \\
Vol. 7 & 1.22 & 0.29 & 4.36 & 2.09 & 6.00 & 1.10 \\
Vol. 8 & 1.37 & 0.42 & 4.12 & 1.36 & 10.31 & 2.55 \\
Vol. 9 & 1.32 & 0.24 & 6.67 & 1.49 & 14.07 & 1.63 \\
\hline
\end{tabular}

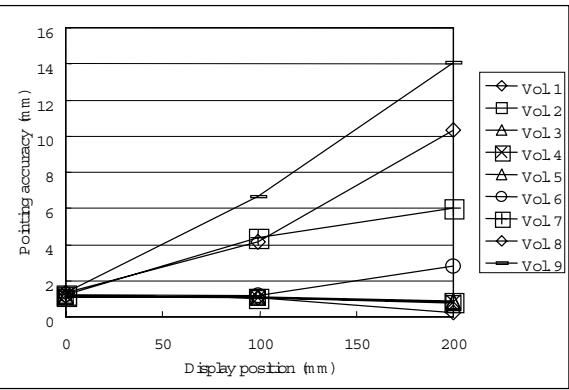

Fig.7. Graph of the data in Table 4 (sorted by distance from the display).

The above experiments verify the pointing accuracy of this system. As a phantom experiment using this system, we used the MRI volume of a volunteer's head as overlay. Fig.8 shows the result. A floating 2-D re-slice image of MRI is observed superimposed on the patient's head. Fig. 7 (R.) shows the image of the eyeballs, and confirms the position of the eyes three-dimensionally.
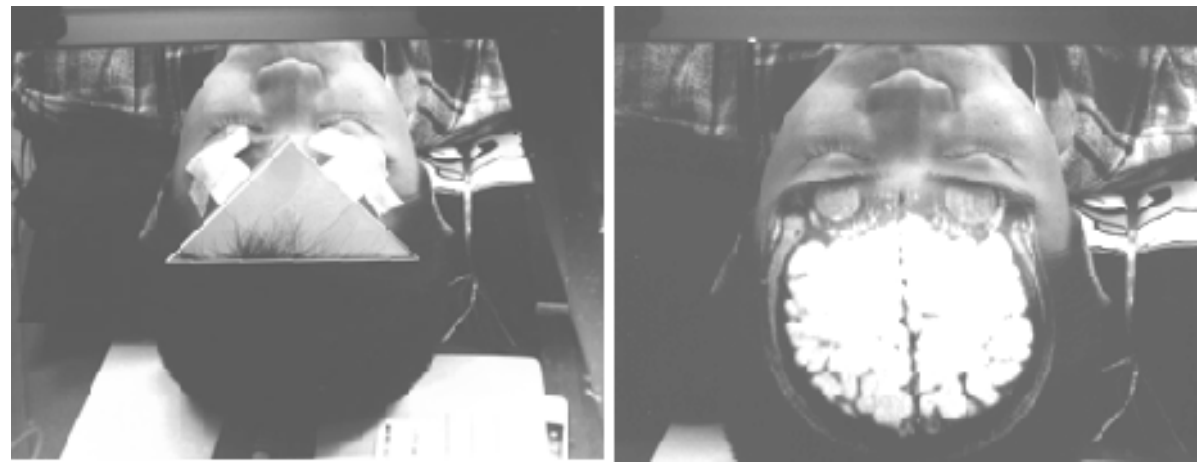

Fig.8. Image overlay experiment Left: adjusting the triangle marker to obtain registration. Right: the 2-D slice image is floating, with accurate depth perception.

\section{Discussion}

In this system, we used a simple raw intersection image, which is familiar to surgeons. In the future, we could also apply more useful pre-acquired data, such as 3$\mathrm{D}$ organ models and structural information on blood vessels. The concept of using a 3-D image visualized in a half-silvered mirror is not an original idea, and similar research is presented in references $1,2,3,4,8$ and 9 . The difference in this system lies in the use of a simple system to create the 3-D image, which would be useful in extending the expression of the 3-D image. This system would be very useful to use 
in real-time image updating, such as X-ray computerized tomography (CT) or interventional magnetic resonance (MR) systems. It will solve the problems of deformation of organs inside the patient.

The registration method we applied in this paper is simple and requires a minimum set of fiducial markers to obtain 3-D registration. Evaluation experiments indicate that this registration system has potential subjective errors that will depend on the human observer's depth perception. Each observer's eyes differ. To solve this problem, we could add more markers or use another method, such as optical tracking, 3-D-position sensor, or arm-type digitizer, and we consider these special components are not convenient for surgical use.

Thus, our research group is also now developing a new 3-D display using integral photography [10]. By mounting this new 3-D display, we will have real-time 3-D images in the operating theater in the near future.

\section{Conclusion}

A 2-D sliced medical image overlay display with accurate depth perception is described. Real depth perception, simple registration, and the capability to be used in the operating theater are presented. The accuracy results indicate that it is useful enough to apply in clinical situations considering the resolution of the pre-acquired images.

\section{References}

[1] R. Taylor, et al., Computer Integrated Surgery - Technology and Clinical Application - , MIT Press, 1995

[2] D.T. Gering, et al., An Integrated Visualization System for Surgical Planning and Guidance Using Image Fusion and Interventional Imaging, Proc. MICCAI99, pp.809-819, 1999

[3] T. Ono, K. Masamune, M. Suzuki, T. Dohi, H. Iseki and K. Takakura, Study on threedimensional surgical support display using enhanced reality, Proc. of 7th annual meeting of Japan Society of Computer Aided Surgery, pp.113-114, 1997 (in Japanese)

[4] M. Blackwell, C. Nikou, A.M. DiGioia and T. Kanade, An Image Overlay System for Medical Data Visualization, MICCAI98, pp.232-240, 1998

[5] W.E.L. Grimson, et al., An Automatic Registration Method for Frameless Stereotaxy, Image Guided Surgery, and Enhanced Reality Visualization, IEEE Trans. Med. Imag., Vol.15, No.2, 1996

[6] Y. Masutani, H. Iseki, et al., NIH Image for surgical operations - Guide in 3-D medical image processing, Nakayama Syoten Press, 1996

[7] NIH Imagae Home page http://rsb.info.nih.gov/nih-image/

[8] Y. Masutani, T. Dohi, Y. Nishi, M. Iwahara, H. Iseki and K. Takakura, VOLUMEGRAPH An integral photography based enhanced reality visualization system, Proc. of CAR'96, p.1051, 1996

[9] Y. Masutani, M. Iwahara, O. Samuta, Y. Nishi, N. Suzuki, M. Suzuki, T. Dohi, H. Iseki and K. Takakura, Development of integral photography based enhanced reality visualization system for surgical support, Proc. of ISCAS'95, pp.16-17, 1995

[10] S. Nakajima, K. Masamune, I. Sakuma, T. Dohi, Development of a 3-D Display System for Surgical Navigation,Trans. of the Institute of Electronics, Information and Communication Eng. D-II, Vol. J83-D-II, No.1, pp.387-395, 2000 